\title{
Spontaneous phase oscillation induced by inertia and time delay
}

\author{
H. Hong, ${ }^{1}$ Gun Sang Jeon, ${ }^{2}$ and M.Y. Choi ${ }^{1}$ \\ ${ }^{1}$ School of Physics and Center for Theoretical Physics, Seoul National University, Seoul 151-747, Korea \\ ${ }^{2}$ Center for Strongly Correlated Materials Research, Seoul National University, Seoul 151-747, Korea
}

\begin{abstract}
We consider a system of coupled oscillators with finite inertia and time-delayed interaction, and investigate the interplay between inertia and delay both analytically and numerically. The phase velocity of the system is examined; revealed in numerical simulations is emergence of spontaneous phase oscillation without external driving, which turns out to be in good agreement with analytical results derived in the strong-coupling limit. Such self-oscillation is found to suppress synchronization and its frequency is observed to decrease with inertia and delay. We obtain the phase diagram, which displays oscillatory and stationary phases in the appropriate regions of the parameters.
\end{abstract}

PACS numbers: 05.45.Xt, 02.30.Ks

Systems of coupled nonlinear oscillators, serving as a prototype model for various oscillatory systems in nature, have been known to exhibit remarkable phenomena of collective synchronization, which have been observed in a variety of physical, biological, and chemical systems [1]. Among them there are systems where finite inertia and time delay are relevant. For example, in a superconducting junction network [2], the capacitance plays the role of inertia while time delay naturally arises in some physical as well as biological systems, where finite time interval is actually required for the transmission of information [3]. This has motivated recent studies of the effects of finite inertia and of time delay on synchronization: In a system with inertia suppression of synchronization and emergence of hysteresis has been observed [ 4 ], whereas a multitude of coherent states with different synchronization frequencies and suppression of the collective frequency have been reported in systems with delay [5]. Note, however, that the effects of the two have been considered separately and independently of each other. The interplay between the two has not been explored in the most general system with both inertia and time delay. For a single oscillator with inertia, it is known that delayed restoring force can destabilize a fixed point and produce oscillation [6]; this raises an interesting question as to how such oscillatory behavior affects collective synchronization in a set of globally coupled oscillators with inertia and delay.

This paper is to investigate the interplay between inertia and retarded interaction and their combined effects on synchronization. We consider a system of globally coupled oscillators, each possessing finite inertia and interacting with others via time-delayed interactions. To explore the interplay, we first examine the temporal behavior of the phase velocity, and obtain a nonvanishing ac component in the absence of external periodic driving. Such spontaneous oscillation is found to suppress synchronization, and its frequency observed to diminish with inertia and delay.

We begin with the set of equations of motion governing the dynamics of $N$ coupled oscillators, the $i$ th of which is described by its phase $\phi_{i}(i=1,2, \ldots, N)$ :

$$
\mu \ddot{\phi}_{i}(t)+\dot{\phi}_{i}(t)+\frac{K}{N} \sum_{j=1}^{N} \sin \left[\phi_{i}(t)-\phi_{j}(t-\tau)\right]=\omega_{i},
$$

where $\mu$ represents the magnitude of (rotational) inertia relative to damping. The third term on the left-hand side denotes the global coupling of strength $K / N$ between oscillators, indicating that each oscillator interacts with others only after the retardation time $\tau$. The term $\omega_{i}$ on the right-hand side denotes the intrinsic frequency of the $i$ th oscillator, and is randomly distributed over the whole oscillators according to $g(\omega)$, which is assumed to be symmetric about $\omega=0$, and concave at $\omega=0$. In the absence of time delay and inertia $(\tau=\mu=0)$, Eq. (11) exactly reduces to the Kuramoto model [7], for which analytical results are available. The system with either time delay or inertia (but not both) also exhibits synchronization for the coupling strength strong enough. The phases are stationary or monotonic in time [4. indicating that the system either relaxes to the minimumenergy configuration or displays constant phase velocity.

Here we investigate the dynamics of phases in the presence of both inertia and delay, first by means of numerical simulations. For convenience, we take the Gaussian distribution with unit variance $\left(\sigma^{2}=1\right)$ for $g(\omega)$ and choose the coupling strength $K=3$, which is larger than the known critical values [4,5]. We thus probe the interplay between inertia and delay in the synchronized state. Equation (11) has been integrated with discrete time steps of $\delta t=0.01$. In computing the order parameter, $N_{t}=10^{6}$ time steps have been used at each run, with the data from the first $9.6 \times 10^{5}$ steps discarded. We have varied both $\delta t$ and $N_{t}$ to verify that the steady state has been attained and performed twenty independent runs with different initial configurations, over which averages have been taken. In this manner, we have computed the power spectrum of the phase velocity:

$$
S \equiv \frac{1}{N} \sum_{j=1}^{N}\left|\psi_{j}(f)\right|^{2}
$$


where $\psi_{j}(f) \equiv \int \dot{\phi}_{j}(t) e^{2 \pi i f t} d t$ is the Fourier component of the phase velocity at frequency $f$.

Figure 1 shows the obtained power spectrum in a system of $N=100$ oscillators, for coupling strength $K=3$ and time delay $\tau=2$ at various inertia values, $\mu=0.4,0.6$, and 0.8. (We have considered the size $N$ up to 3200 , and confirmed the absence of appreciable finitesize effects for $N \gtrsim 100)$. For small inertia $(\mu \lesssim 0.3)$, the power spectrum possesses only the zero-frequency component. Namely, the system displays stationary or monotonic behavior of the phase, like the one with delay only [5]. When the inertia is raised beyond 0.3 , on the other hand, several peaks develop at nonzero fundamental and harmonic frequencies, and the oscillatory phase emerges. As $\mu$ is increased further, those peaks shift to the lower frequency sides, eventually disappearing for $\mu \gtrsim 1.0$. We have examined such self-oscillatory behavior for various values of $K, \tau$, and $\mu$, and observed that the oscillation frequency $f_{0}$ decreases with both inertia and delay, as shown in Fig. 2 for $\tau=1.8,2.0$, and 2.2 .

The resulting phase boundaries in the plane of $(\mu, \tau)$, discriminating the oscillatory state and the stationary one, are displayed in Fig. 3, where the coexistence of the two states is also identified in the region between solid squares and open ones. In the coexistence region, there exist a multitude of solutions, giving stationary or oscillatory behavior depending on the initial conditions. To explore this, we examine the behavior of the order parameter $\Delta \equiv N^{-1}\left|\sum_{j} \exp \left(i \phi_{j}\right)\right|$ with the inertia. Figure 4(a) shows the order parameter computed via numerical simulations, as the inertia is varied between 0.1 and 1.2 with the increment of $\delta \mu=0.01$. We have first decreased the inertia from 1.2 to 0.1 , which yields the steplike structure consisting of the solid circles in Fig. 4(a). Upon increasing the inertia from given value, however, the order parameter does not reverse the path along the solid circles; instead it exhibits continuous dependence on the inertia, eventually following the open circles. In this manner a multitude of solutions or "bands" have been found, as shown in Fig. 4(a). Note that there are two kinds in shape, flat bands and bent ones. As the behavior of the phase velocity is examined, the system is stationary in the flat bands, existing for small inertia $(\mu \lesssim 0.3)$ and for large inertia $(\mu \gtrsim 1.1)$. In the bent bands (for $0.3 \lesssim \mu \lesssim 0.9$ ), on the other hand, the system displays phase oscillations. Accordingly, in the range $0.9 \lesssim \mu \lesssim 1.1$, where two kinds of bands coexist, either the oscillatory phase or the stationary one is expected to appear depending on the initial conditions. This coexistence range of inertia (for $\tau=2$ ) is indeed consistent with Fig. 3.

To understand analytically these numerical results, we first divide the population of the oscillators into two groups: the synchronized group $(S)$ and the desynchronized one $(D)$, and take the ansatz $\phi_{i}=\phi_{i}^{0}+\omega_{i} t$ for $D$ and

$$
\phi_{i}=\phi_{i}^{0}+A \sin (\Omega t+\alpha)
$$

for $S$. In view of the symmetry of $g(\omega)$, we consider the dc component $\phi_{i}^{0}$ to be constant. Results of numerical simulations also manifest that near the phase boundary the ac component is much smaller than the dc component, allowing the expansion in terms of the amplitude A. Upon substituting Eq. (3) into Eq. (1), we thus obtain for the oscillators in $S$, to the order of $A^{2}$,

$$
\begin{aligned}
\omega_{i} & =\frac{K}{N} \sum_{j}^{(S)} \sin \left(\phi_{i}^{0}-\phi_{j}^{0}\right)\left(1-A^{2} \sin ^{2} \frac{\Omega \tau}{2}\right) \\
& -\left[\mu \Omega^{2}-\frac{2 K}{N} \sum_{j}^{(S)} \cos \left(\phi_{i}^{0}-\phi_{j}^{0}\right) \sin ^{2} \frac{\Omega \tau}{2}\right] A \sin (\Omega t+\alpha) \\
& +\left[\Omega+\frac{K}{N} \sum_{j}^{(S)} \cos \left(\phi_{i}^{0}-\phi_{j}^{0}\right) \sin \Omega \tau\right] A \cos (\Omega t+\alpha), \quad(4)
\end{aligned}
$$

where the summations run over $S$. Oscillators in $D$, the fraction of which over the whole is negligibly small, $O\left(\mathrm{e}^{-(K \Delta)^{2} / 2 \sigma^{2}}\right)$, turn out not to contribute to the harmonics in $\Omega$. A similar expansion for $D$ leads to trivial equations.

The zeroth harmonics in Eq. (4) leads to

$$
\frac{1}{N} \sum_{j}^{(S)} \sin \left(\phi_{i}^{0}-\phi_{j}^{0}\right)=\frac{\omega_{i}}{K}\left[1+A^{2} \sin ^{2}(\Omega \tau / 2)\right]
$$

or, in terms of the order parameter,

$$
\Delta^{2}=1-\frac{\sigma^{2}}{K^{2}}\left[1+2 A^{2} \sin ^{2}(\Omega \tau / 2)\right]
$$

where $\sigma^{2}$ is the variance of the distribution $g(\omega)$ and the thermodynamic limit $(N \rightarrow \infty)$ together with the strong coupling limit $\left(K \gg K_{c}\right)$ has been taken. It is shown in Eq. (6) that the appearance of oscillation $(A \neq 0)$ reduces $\Delta$, suppressing synchronization.

We next consider the first harmonics in Eq. (4), and obtain [9]

$$
\begin{aligned}
K \Delta^{2}(1-\cos \Omega \tau) & =\mu \Omega^{2}, \\
K \Delta^{2} \sin \Omega \tau & =-\Omega,
\end{aligned}
$$

which yields only the trivial solution $\Omega=0$ in the absence of either inertia or delay. It is thus concluded that the (sustained) spontaneous oscillation requires both inertia and delay. Elimination of $\Delta$ in Eq. (7) leads to the equation for the oscillating frequency $\Omega$ :

$$
\Omega=-\frac{1}{\mu} \tan \frac{\Omega \tau}{2},
$$

the nonzero solution $(\Omega \neq 0)$ of which may be searched in the range $\pi<\Omega \tau<2 \pi$. Equation (7) together with Eq. (8) gives $\Delta^{2}$ in the form 


$$
\Delta^{2}=\frac{1}{2 K \mu}\left(1+\mu^{2} \Omega^{2}\right) .
$$

Note in Eq. (6) that $\Delta$ approaches unity as $\sigma / K$ is reduced to zero $(\sigma / K \rightarrow 0)$. In particular Eq. (6) gives the range $0 \leq \Delta^{2} \leq 1-\sigma^{2} / K^{2}$, where the upper bound of $\Delta^{2}$ determines the boundary between stationary and oscillatory states. Accordingly, on the boundary Eq. (9) reads

$$
\Omega=\sqrt{\frac{2 K}{\mu}\left(1-\frac{\sigma^{2}}{K^{2}}\right)-1},
$$

which in turn yields the boundary

$$
\begin{aligned}
\tau & =2\left[\frac{2 K}{\mu}\left(1-\frac{\sigma^{2}}{K^{2}}\right)-\frac{1}{\mu^{2}}\right]^{-1 / 2} \\
& \times\left[\pi-\arctan \sqrt{2 K \mu\left(1-\frac{\sigma^{2}}{K^{2}}\right)-1}\right] .
\end{aligned}
$$

It can be observed that the oscillatory state appears only for $\mu \geq \mu_{c} \equiv\left[2 K\left(1-\sigma^{2} / K^{2}\right)\right]^{-1}$. The critical value $\mu_{c}$ decreases, allowing larger regions of the oscillatory state, as the coupling strength $K$ is raised and as the variance $\sigma^{2}$ is reduced. The order parameter $\Delta$ obtained from Eq. (7) for $K=3$ and $\tau=2$ is plotted by the broken curve in Fig. 4 (b), manifesting good agreement with the numerical result. The tiny discrepancy of $O\left(10^{-2}\right)$ between the two curves can be attributed to the truncation of higher-order terms in Eq. (4).

Solving Eq. (8) in the appropriate regime, we obtain the oscillation frequency as a function of inertia and delay. Figure 5(a) shows the behavior of the fundamental frequency $\Omega / 2 \pi$ on the plane of inertia and delay. For comparison, the dependence on inertia for given amount of delay $\tau=1.8,2.0$, and 2.2 is shown in Fig. 5(b), together with the corresponding numerical data (previously plotted in Fig. 2). Note the good agreement between the analytical results, obtained from Eq. (8), and those from numerical simulations. It is manifested that the oscillation frequency in general decreases with the amount of delay and inertia.

The phase diagram in the three-dimensional space of $(\mu, \tau, K)$ is displayed in Fig. 6(a), where the boundary surface separates the oscillatory state from the stationary one. Figure 6(b) exhibits the phase boundaries on the plane of inertia and delay for several values of the coupling strength. Also shown are the data obtained from numerical simulations for $K=3$ (see Fig. 3), again demonstrating good agreement of the analytical results based on Eq. 111 with the simulation results. It is further observed that the region of the oscillatory state grows with the coupling strength. Note, however, that the analytical approach, focusing on the existence of a nonzero solution for $\Omega$, is not able to discern the coexistence of oscillatory and stationary phases from the oscillatory phase. Thus the numerical data represented by solid squares in Fig. 3 have no counterpart in the analytical results shown in Fig. 6.

In summary, we have studied the synchronization phenomena in a system of coupled oscillators, each possessing finite inertia and interacting with others via timedelayed interactions. The interplay between inertia and time delay has been investigated in the temporal behavior of the phase velocity, which reveals the emergence of (sustained) spontaneous oscillation in the absence of external periodic driving. We have also obtained the phase diagram, which displays oscillatory and stationary states in the appropriate regions of the three-dimensional space consisting of the inertia, delay, and coupling strength.

We acknowledge the hospitality from Korea Institute for Advanced Study, where part of this work was performed, and the partial support from the BK21 Project.

[1] For a list of references, see A.T. Winfree, The Geometry of Biological Time (Springer-Verlag, New York, 1980); Y. Kuramoto, Chemical Oscillations, Waves, and Turbulence (Springer-Verlag, Berlin, 1984).

[2] K. Park and M.Y. Choi, Phys. Rev. B 56, 387 (1997).

[3] D.V. Ramana Reddy, A. Sen, and G.L. Johnston, Phys. Rev. Lett. 85, 3381 (2000).

[4] H.-A. Tanaka, A.J. Lichtenberg, and S. Oishi, Phys. Rev. Lett. 78, 2104 (1997); H. Hong, M.Y. Choi, J. Yi, and K.S. Soh, Phys. Rev. E 59, 353 (1999); H. Hong, M.Y. Choi, B.-G. Yoon, K. Park, and K.-S. Soh, J. Phys. A 32, L9 (1999); J.A. Acebron, L.L. Bonilla, and R. Spigler, Phys. Rev. E 62, 3437 (2000); H. Hong and M.Y. Choi, ibid. 62, 6462 (2000).

[5] E. Niebur, H.G. Schuster, and D.M. Kammen, Phys. Rev. Lett. 67, 2753 (1991); Y. Nakamura, F. Tominaga, and T. Munakata, Phys. Rev. E 49, 4849 (1994); S. Kim, S.H. Park, and C.S. Ryu, Phys. Rev. Lett. 79, 2911 (1997); M.K.S. Yeung and S.H. Strogatz, Phys. Rev. Lett. 82, 648 (1999); M.Y. Choi, H. J. Kim, D. Kim, and H. Hong, Phys. Rev. E 61, 371 (2000).

[6] See, e.g., N. MacDonald, Biological Delay Systems: Linear Stability Theory (Cambridge University Press, Cambridge, 1989).

[7] Y. Kuramoto and I. Nishikawa, J. Stat. Phys. 49, 569 (1987); H. Daido, Prog. Theor. Phys. 77, 622 (1987); Phys. Rev. Lett. 68, 1073 (1992).

[8] Here higher harmonics have much smaller amplitudes and are disregarded.

[9] We replace the quantity $N^{-1} \sum_{j}^{(S)} \cos \left(\phi_{i}^{0}-\phi_{j}^{0}\right)$ by its mean value $\Delta^{2}$. To be precise, it varies with $i$ in $O\left(\sigma^{2} / K^{2}\right)$; thus the ansatz in Eq. (3) is exact in the limit $K \rightarrow \infty$. Such rather small fluctuations as well as the good agreement between the analytical and numerical results indicate that the ansatz still provides a good approximation for large but finite $K$. 


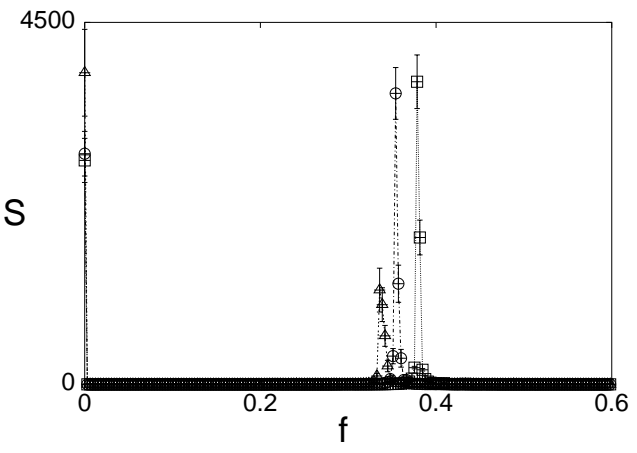

FIG. 1. Power spectrum of the phase velocity (in arbitrary units) for coupling strength $K=3$, time delay $\tau=2$, and inertia $\mu=0.4$ (squares), 0.6 (circles), 0.8 (triangles). Only the fundamental peaks are displayed. Error bars have been estimated by the standard deviation and lines are merely guides to the eye.

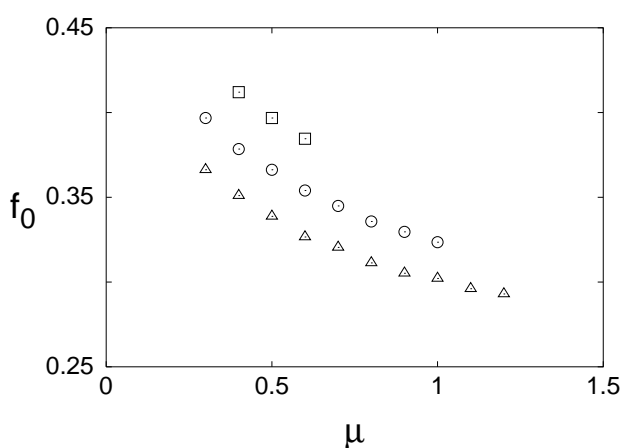

FIG. 2. Oscillation frequency $f_{0}$ for $K=3$ is shown to decrease with inertia $\mu$ at various amounts of delay: $\tau=1.8$ (squares), 2.0 (circles), and 2.2 (triangles). The sizes of the error bars estimated by the standard deviation are about the same as those of the symbols.

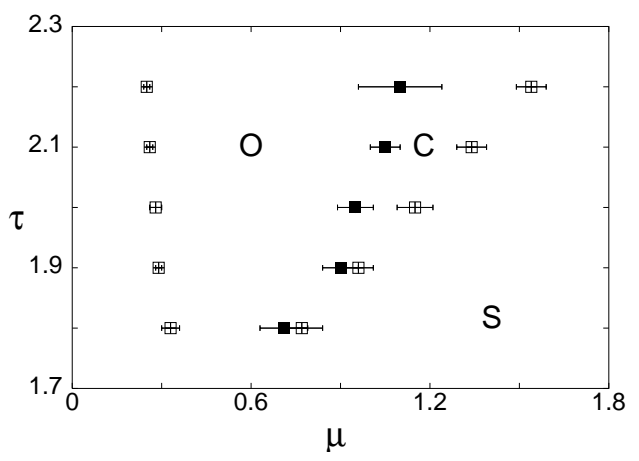

FIG. 3. Phase boundaries between the oscillatory phase $(\mathrm{O})$ and the stationary one $(\mathrm{S})$ are exhibited in the plane of $(\mu, \tau)$ for $K=3$. Also displayed is the boundary separating the coexistence region $(\mathrm{C})$. The data represented by open and solid squares have been obtained from numerical simulations with five different configurations; error bars have been estimated by the range of the obtained values.

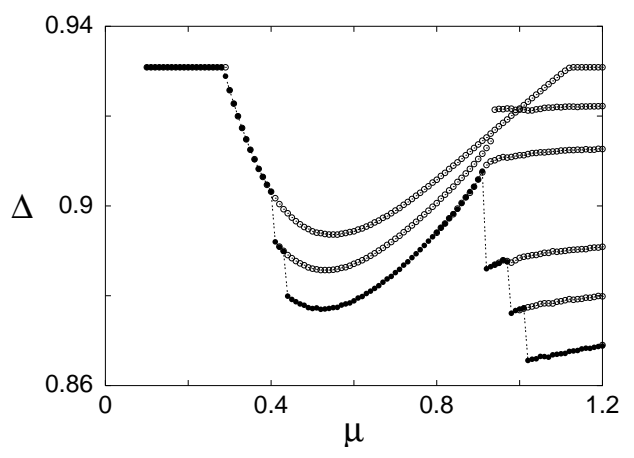

(a)

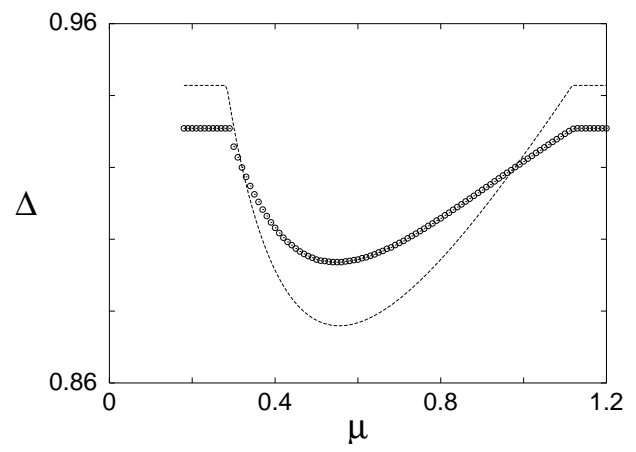

(b)

FIG. 4. (a) Behavior of the order parameter with inertia for $K=3$ and $\tau=2$, obtained numerically. (b) Comparison with the analytical results (broken curve).

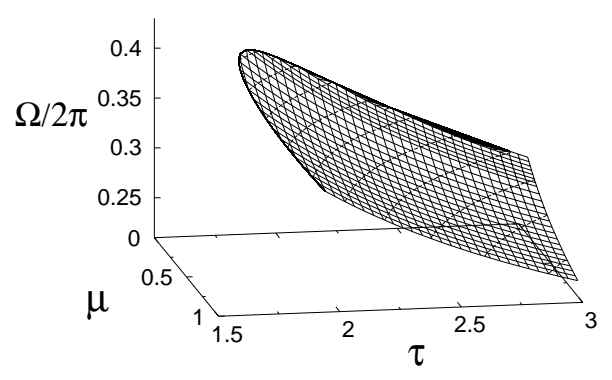

(a)

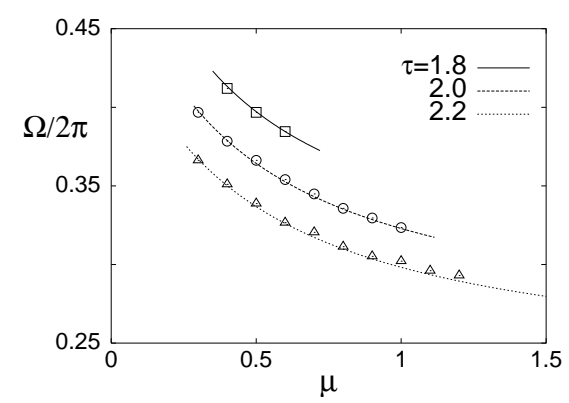

(b) 
FIG. 5. Behavior of the fundamental frequency $\Omega / 2 \pi$ for $K=3$ (a) in the $(\mu, \tau)$ plane; (b) versus $\mu$ for $\tau=1.8$, 2.0 , and 2.2 , obtained analytically. The data from numerical simulations, shown in Fig. 2, are also plotted for comparison.

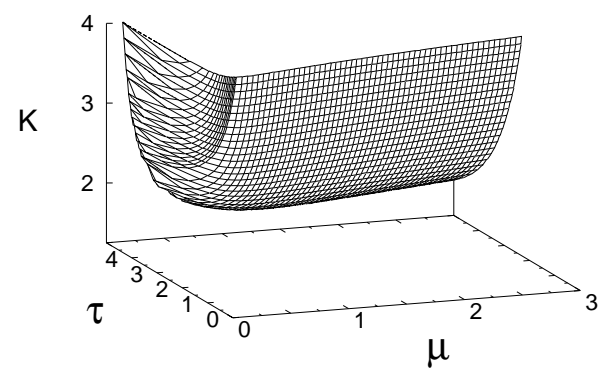

(a)

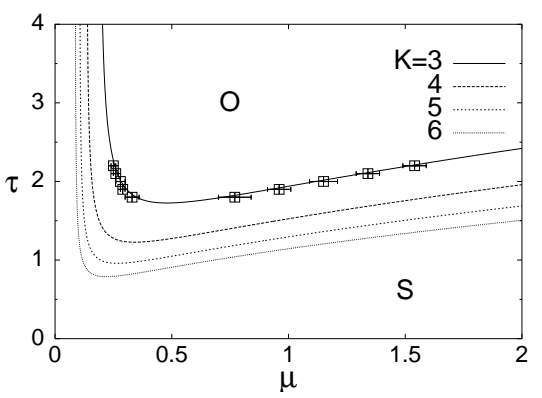

(b)

FIG. 6. Phase diagram (a) in the three-dimensional $(\mu, \tau, K)$ space; (b) in the $(\mu, \tau)$ plane for several values of the coupling strength, obtained analytically. The data from numerical simulations for $K=3$, shown in Fig. 3, are also plotted for comparison. 